\title{
Avaliação dos atendimentos endodônticos em um Centro de Especialidades Odontológicas em Porto Alegre, RS
}

\author{
Guilherme Daniel Dörr*, Fabiana Soares Grecca*, Jessye Melgarejo do Amaral Giordani** \\ * Faculdade de Odontologia da Universidade Federal do Rio Grande do Sul, \\ Porto Alegre, RS \\ ** Faculdade de Odontologia da Universidade Federal de Santa Maria, Santa \\ Maria, RS
}

\begin{abstract}
RESUMO
O presente estudo tem por objetivo descrever o perfil dos atendimentos endodônticos do Centro de Especialidades Odontológicas Bom Jesus. Trata-se de um estudo observacional transversal descritivo, utilizando prontuários clínicos de todos os usuários atendidos no período de 2012 e 2013, na especialidade de endodontia, oriundos de dois distritos sanitários de Porto Alegre. Foram realizadas análises das frequências absolutas e relativas das variáveis relacionadas aos usuários e aos procedimentos, estratificando-as em aspectos gerais e por distrito sanitário. A maioria dos usuários $(70,2 \%)$ era do sexo feminino, com idade até 19 anos $(22,2 \%)$ e provenientes do distrito Partenon $(68,8 \%)$. Os primeiros molares apresentaram maior demanda $(38,5 \%)$ seguido dos pré-molares $(22,7 \%)$, dos dentes da arcada superior $(56,7 \%)$ e com indicação de necropulpectomia $(84,8 \%)$. Em conclusão, foi possível identificar o distrito sanitário de maior demanda, bem como características demográficas e clínicas dos usuários, auxiliando no planejamento e resolutividade das ações odontológicas no Sistema Único de Saúde e na formação dos estudantes de odontologia mais próxima do cotidiano a ser vivenciado no exercício profissional.
\end{abstract}

Descritores: Serviços de Saúde Bucal. Atenção Secundária à Saúde. Especialidades Odontológicas. Endodontia. 


\section{INTRODUÇÃO}

No Sistema Único de Saúde (SUS), ordenador da formação em saúde, as redes de atenção à saúde representam papel importante como campos de estágios curriculares dos estudantes de Odontologia, pois os projetos pedagógicos orientados por estratégias de ensinoaprendizagem que respeitam a integração ensinoserviço-comunidade proporcionam formação mais próxima do cotidiano a ser vivenciado no exercício profissional. Nesse sentido, a aproximação da universidade ao profissional do serviço de saúde, apoiando-o nas ações que desenvolve em conjunto com os estudantes é compreendida como necessária para a efetivação dos princípios do SUS ${ }^{1}$.

A organização do sistema de referência e contrarreferência é um dos fatores mais importantes para efetivação da atenção integral a saúde $^{2}$. A referência é representada pelo fluxo, por meio de encaminhamento do paciente com necessidades de saúde complexas, para centros de atenção especializada. O fluxo contrário, ou seja, o retorno do paciente ao nível da atenção básica, próxima de sua residência, é denominado contrarreferência ${ }^{3}$.

Uma das formas de organizar o sistema de referência e contrarreferência nos municípios brasileiros é através da divisão da cidade em distritos sanitários. É de suma importância estudar e reorganizar os serviços nos distritos sanitários, considerando uma base populacional definida, bem como seu perfil epidemiológico, buscando a melhora da efetividade na prestação da atenção à saúde. O território do distrito sanitário pode ser caracterizado por uma determinada população, que vive em um espaço específico, com seus problemas de saúde, interagindo com todos os componentes da rede. Além disso, é um espaço político e administrativo dentro do sistema de saúde ${ }^{4}$. O que embasa a delimitação do território no distrito sanitário é o planejamento urbano de uma forma administrativo-assistencial. Uma vez instituído o distrito sanitário neste local, como organização administrativa, ele terá certo grau de autonomia, voltando-se para as mudanças necessárias para atenção à saúde ${ }^{5}$.

É de grande relevância avaliar as informações de acesso e demanda reprimida para especialidades, bem como o percentual de faltas, abandono de tratamento pelo paciente $\mathrm{e}$ resolutividade na especialidade, pois estes dados podem refletir a qualidade da atenção básica na efetivação da integralidade, além do seu papel de coordenação do cuidado e acompanhamento longitudinal através da rede de serviços ${ }^{6}$.

$\mathrm{Na}$ área de saúde bucal, quem presta o serviço na atenção básica são as equipes de saúde bucal (ESB), que podem ou não estar vinculadas à Estratégia Saúde da Família. Estas ESB têm a possibilidade de referenciar os usuários dos serviços de saúde bucal para outro nível de atenção (nível secundário), onde os Centros de Especialidades Odontológicas (CEO) fazem o atendimento especializado e trabalham na lógica da referência e contrarreferência, integrando os serviços de saúde bucal dos diferentes níveis de atenção ${ }^{7}$. Os CEO estão sendo implantados em todas as regiões do Brasil, como uma das principais metas da Política Nacional de Saúde Bucal, que tem como um de seus objetivos, expandir e melhorar a oferta da atenção secundária em Odontologia, garantindo assim uma efetivação da integralidade à saúde ${ }^{8}$.

Os CEO são classificados em três tipos: tipo I (três cadeiras odontológicas), tipo II (quatro a seis cadeiras) e tipo III (acima de sete cadeiras). A implantação de um CEO funciona por meio de parcerias entre estados, municípios e Governo Federal. O Ministério da Saúde (MS) repassa um incentivo para implantação (construção, reforma, aquisição de equipamentos odontológicos) e custeio dos CEO. O CEO tipo I recebe $\mathrm{R} \$ 60 \mathrm{mil}$ para implantação e $R \$ 8.250,00$ para custeio mensal. Já o CEO tipo II recebe $\mathrm{R} \$ 75$ mil para implantação e R \$ 11 mil para custeio mensal. Enquanto que para o CEO tipo III são repassados R\$ 120 mil para implantação e R \$ 19.250,00 para custeio mensal. Para receber estes recursos, todos os CEO devem obedecer algumas exigências do 
MS, entre elas a meta relativa à quantidade de procedimentos ${ }^{9}$.

Os CEO ainda estão em fase de desenvolvimento no Brasil, tanto no que tange à quantidade e à otimização dos serviços prestados. Problemas vêm sendo relatados na literatura, principalmente no que diz respeito ao cumprimento das metas estabelecidas. Sendo assim, é de suma importância que se tenha um melhor entendimento desta relação entre serviços prestados e perfil de usuários. Havendo mais subsídios, é possível enfrentar as dificuldades apontadas de forma mais consistente ${ }^{10-12}$.

Diante deste contexto, o estudo objetiva descrever o perfil dos pacientes e dos procedimentos da especialidade de endodontia do CEO da Vila Bom Jesus, oriundos de duas gerências distritais, na cidade de Porto Alegre, RS, no período de 2012 e 2013.

\section{MÉTODOS}

Trata-se de um estudo observacional transversal descritivo, onde foram analisados prontuários clínicos de todos os usuários atendidos no período de 2012 e 2013, na especialidade de endodontia do CEO do Centro de Saúde Bom Jesus, oriundos das unidades de saúde dos distritos Leste-Nordeste e Partenon-Lomba do Pinheiro, da cidade de Porto Alegre, Rio Grande do Sul. Este serviço de saúde recebe usuários oriundos de um distrito sanitário onde os estudantes de graduação em Odontologia da Universidade Federal do Rio Grande do Sul realizam os estágios curriculares nas unidades de atenção primária à saúde, na perspectiva das redes de atenção em saúde e na utilização de dispositivos de referência e contrarreferência como parte do processo de produção da saúde, integrando os alunos com os profissionais de atenção primária e do CEO.

$\mathrm{O}$ projeto de pesquisa utilizou dados secundários oriundos de prontuários e foi aprovado pela Comissão de Pesquisa da Faculdade de Odontologia da Universidade Federal do Rio Grande do Sul sob o número 28013, seguindo as condições estabelecidas na Resolução 196/96 do
Conselho Nacional de Saúde (CNS).

No município de Porto Alegre, o território e a sua população (1.409.351 habitantes) estão distribuídos em 17 Distritos Sanitários (DS), onde para cada distrito sanitário foram definidos os limites geográficos e sua população, no intuito de regionalizar o conjunto de serviços a serem ofertados. As Gerências Distritais (GD), que são estruturas administrativas e também espaços de discussão e prática, abrangem o território de um ou mais DS, sendo um total de oito GD, com população entre 95 e 200 mil habitantes ${ }^{13}$. Os serviços de saúde bucal na atenção básica são ofertados em 117 unidades de saúde, sendo 45 Unidades Básicas de Saúde e 72 Unidades de Saúde da Família. Na atenção especializada, o município de Porto Alegre possui ao todo seis $\mathrm{CEO}$, que dividem responsabilidades sobre as oito gerências distritais. O CEO do Centro de Saúde Bom Jesus, objeto de estudo deste trabalho, recebe usuários das unidades de saúde das gerências distritais Leste-Nordeste e Partenon-Lomba do Pinheiro e atua com carga horária de 50 horas semanais. A Gerência Distrital Partenon-Lomba do Pinheiro abrange uma população de 173.706 pessoas, enquanto que a Gerência Distrital LesteNordeste possui 151.295 pessoas, totalizando 325.001 pessoas que estão dentro da área de abrangência do CEO Bom Jesus. Ao todo, são 30 unidades de saúde que referenciam usuários para a especialidade de Endodontia do CEO Bom Jesus, sendo 11 unidades da gerência distrital LesteNordeste e 19 unidades da gerência distrital Partenon-Lomba do Pinheiro ${ }^{14}$.

A coleta de dados teve início no ano de 2014, após a aprovação pelo Comitê de Ética em Pesquisa e utilizou informações dos prontuários dos usuários atendidos no CEO do Centro de Saúde Bom Jesus avaliando-se as fichas clínicas com os questionários de saúde e os termos de consentimento livre e esclarecido, além de exames de imagem dos casos. Para a extração dos dados dos prontuários, foi utilizado um instrumento padronizado estruturado e pré-testado, sendo realizada checagem piloto após as primeiras cole- 
tas. Para compor o instrumento de coleta de dados, todos os procedimentos contidos no Manual de Especialidades em Saúde Bucal do Ministério da Saúde foram utilizados e acrescidos de outras informações conforme descrição das variáveis ${ }^{15}$. Os tratamentos endodônticos realizados no CEO Bom Jesus foram executados por dois profissionais, sendo um especialista na área e outro com curso de especialização em andamento, seguindo o protocolo mesmo clínico.

Como os atendimentos são registrados por procedimentos, houve a necessidade da busca de informações divididas em duas categorias: variáveis relativas às pessoas (usuários atendidos no serviço de endodontia do CEO); e variáveis relativas aos procedimentos (dentes).

Para as informações relativas às pessoas e aos procedimentos foram utilizadas as variáveis que constam do quadro 1.

Para a análise estatística foi utilizado o software SPSS versão 18. Foram realizadas as análises das frequências absolutas e relativas das variáveis, estratificando-as em aspectos gerais (relativo à amostra total) e por distrito sanitário (Partenon-Lomba do Pinheiro e Leste-Nordeste). Todas as análises foram subdivididas em características das pessoas e características dos procedimentos.

Quadro 1. Variáveis coletadas para o estudo.

\author{
Relativas às pessoas \\ Distrito sanitário: Partenon / Nordeste) \\ Idade (coletada de forma contínua e categorizada em faixas etárias): até 18 anos / 19 a 24 / 25 a 34 / 35 a 44 / \\ 45 a 54 / 55 a 64 / 65 anos ou mais \\ Sexo: masculino / feminino \\ Período do atendimento: ano e semestre da última consulta no CEO para o atendimento \\ Condição sistêmica: sim / não, para refere hipertensão, refere diabetes, refere hepatite e nega uso de \\ medicamentos
}

\title{
Relativas aos procedimentos
}

Dente: incisivo / canino / pré-molar $/ 1^{\circ}$ molar $/ 2^{\circ}$ molar $/ 3^{\circ}$ molar

Arcada dentária: superior / inferior

Tipo de tratamento: necropulpectomia / biopulpectomia / retratamento / outros (tratamentos pouco comuns no CEO Bom Jesus, tais como apicificação, apicigênese, pulpotomia, capeamento pulpar direto e indireto, cirurgia apical)

Dor dentária durante o período de tratamento endodôntico: sim / não (com base na ficha clínica odontológica de cada usuário)

Necessidade de adequação do dente antes do início do tratamento: sim / não (referência: critérios clínicos de encaminhamento para endodontia do Protocolo de Atenção em Saúde Bucal de Porto Alegre ${ }^{16}$ )

Impossibilidade de realização do tratamento endodôntico: sim / não (referência: critérios de exclusão para endodontia no CEO presentes no Protocolo de Atenção em Saúde Bucal de Porto Alegre ${ }^{16}$ )

Número de sessões necessárias para a conclusão do tratamento endodôntico: uma sessão / duas sessões / três sessões / quatro ou mais (as consultas realizadas na atenção básica não foram contabilizadas; como protocolo, a primeira consulta no CEO Bom Jesus, em geral é realizada apenas a anamnese, exame clínico, radiografia inicial, e apenas em uma segunda consulta, o tratamento endodôntico propriamente dito inicia, com exceção para os casos em que o profissional optou por iniciar na primeira consulta por motivos de organização de agenda e ou sintomatologia dolorosa do paciente)

Conclusão do tratamento: sim / não / não necessitou (dentes que não necessitavam de tratamento endodôntico, ou que não estavam mais em boca)

Abandono do tratamento: sim / não / não necessitou (dentes que não necessitavam de tratamento endodôntico, ou que não estavam mais em boca) 


\section{RESULTADOS}

Foram analisados prontuários de 487 usuários, totalizando 538 dentes. Em relação aos usuários, 68,8\% foram referenciados de unidades do distrito Partenon-Lomba do Pinheiro e 31,2\% de unidades da gerência Leste-Nordeste. A maioria $(70,2 \%)$ era do sexo feminino, com idade até 19 anos (22,2\%), não referindo hipertensão $(80,9 \%)$, diabetes $(93,2 \%)$, hepatite $(95,9 \%)$ ou uso contínuo de algum tipo de medicamento $(62,0 \%)$ (tabela 1).

Tabela 1- Descrição da amostra geral e estratificada em distritos sanitários segundo características dos pacientes $(\mathrm{n}=487)$.

\begin{tabular}{|c|c|c|c|}
\hline \multirow[b]{2}{*}{ Variável } & \multirow[b]{2}{*}{$\begin{array}{l}\text { Geral } \\
\text { n }(\%)\end{array}$} & \multicolumn{2}{|c|}{ Distrito Sanitário } \\
\hline & & $\begin{array}{c}\text { Nordeste } \\
\text { n }(\%)\end{array}$ & $\begin{array}{c}\text { Partenon } \\
\text { n (\%) }\end{array}$ \\
\hline \multicolumn{4}{|l|}{ Distrito Sanitário } \\
\hline Partenon/Lomba & $335(68,8)$ & - & - \\
\hline Nordeste & $152(31,2)$ & - & - \\
\hline \multicolumn{4}{|l|}{ Sexo } \\
\hline Feminino & $342(70,2)$ & $103(67,8)$ & $239(71,3)$ \\
\hline Masculino & $145(29,8)$ & $49(32,2)$ & $96(28,7)$ \\
\hline \multicolumn{4}{|l|}{ Faixa etária } \\
\hline Até 18 anos & $108(22,2)$ & $34(22,7)$ & $74(22)$ \\
\hline De 19 a 24 anos & $47(9,7)$ & $15(10)$ & $32(9,5)$ \\
\hline De 25 a 34 anos & $88(18,1)$ & $20(13,3)$ & $68(20,2)$ \\
\hline De 35 a 44 anos & $94(19,3)$ & $28(18,7)$ & $66(19,6)$ \\
\hline De 45 a 54 anos & $79(16,2)$ & $26(17,3)$ & $53(15,7)$ \\
\hline De 55 a 64 anos & $55(11,3)$ & $19(12,7)$ & $36(10,7)$ \\
\hline 65 anos ou mais & $16(3,3)$ & $8(5,3)$ & $8(2,4)$ \\
\hline \multicolumn{4}{|l|}{ Período } \\
\hline $1^{\circ}$ semestre de 2012 & $94(19,3)$ & $29(19,3)$ & $65(19,3)$ \\
\hline $2^{\circ}$ semestre de 2012 & $174(35,7)$ & $43(28,7)$ & $131(38,9)$ \\
\hline $1^{\circ}$ semestre de 2013 & $128(26,3)$ & $37(24,7)$ & $91(27)$ \\
\hline $2^{\circ}$ semestre de 2013 & $91(18,7)$ & $41(27,3)$ & $50(14,8)$ \\
\hline \multicolumn{4}{|l|}{ Hipertensos } \\
\hline Sim & $93(19,1)$ & $37(24,7)$ & $56(16,6)$ \\
\hline Não & $394(80,9)$ & $113(75,3)$ & $281(83,4)$ \\
\hline \multicolumn{4}{|l|}{ Diabéticos } \\
\hline Sim & $33(6,8)$ & $13(8,7)$ & $20(5,9)$ \\
\hline Não & $454(93,2)$ & $137(91,3)$ & $317(94,1)$ \\
\hline \multicolumn{4}{|l|}{ Hepatite } \\
\hline Sim & $20(4,1)$ & $8(5,3)$ & $12(3,6)$ \\
\hline Não & $467(95,9)$ & $142(94,7)$ & $325(96,4)$ \\
\hline \multicolumn{4}{|l|}{ Uso de medicamentos } \\
\hline Sim & $185(38)$ & $57(38)$ & $128(38)$ \\
\hline Não & $302(62)$ & $93(62)$ & $209(62)$ \\
\hline
\end{tabular}


Segundo as características dos procedimentos realizados, os primeiros molares foram os dentes de maior demanda $(38,5 \%)$ seguido dos pré-molares $(22,7 \%)$. Os tratamentos foram realizados, em sua maioria, em dentes da arcada superior $(56,7 \%)$, com indicação de necropulpectomia $(84,8 \%)$ e em até três sessões $(81,5 \%)$. Os dentes que necessitavam de adequação representaram 34,9\% da amostra e 13,8\% deles foram considerados impossíveis de serem tratados. A dor foi relatada em $26 \%$ dos casos e o tratamento foi concluído em $65,8 \%$ dos dentes, sendo que $17,1 \%$ tiveram seu tratamento interrompido por motivo de abandono do usuário. Ainda, 3,3\% dos dentes referenciados não necessitavam de tratamento endodôntico (tabela 2).

Tabela 2 - Descrição da amostra geral e estratificada em distritos sanitários segundo características dos procedimentos $(\mathrm{n}=538)$.

\begin{tabular}{|c|c|c|c|}
\hline \multirow[b]{2}{*}{ Variável } & \multirow[b]{2}{*}{$\begin{array}{l}\text { Geral } \\
\text { n }(\%)\end{array}$} & \multicolumn{2}{|c|}{ Distrito Sanitário } \\
\hline & & $\begin{array}{c}\text { Nordeste } \\
\text { n }(\%)\end{array}$ & $\begin{array}{c}\text { Partenon } \\
\text { n }(\%)\end{array}$ \\
\hline \multicolumn{4}{|l|}{ Dente } \\
\hline Incisivo & $103(19,1)$ & $24(14,4)$ & $79(21,3)$ \\
\hline Canino & $31(5,8)$ & $14(8,4)$ & $17(4,6)$ \\
\hline Pré-molar & $122(22.7)$ & $41(24.6)$ & $81(21.8)$ \\
\hline $1^{\circ}$ molar & $207(38,5)$ & $69(41,3)$ & $138(37,2)$ \\
\hline $2^{\circ}$ molar & $70(13)$ & $16(9,6)$ & $54(14,6)$ \\
\hline $3^{\circ}$ molar & $5(0,9)$ & $3(1,8)$ & $2(0,5)$ \\
\hline \multicolumn{4}{|l|}{ Arcada } \\
\hline Superior & $305(56,7)$ & $87(52,1)$ & $218(58,8)$ \\
\hline Inferior & $233(43,3)$ & $80(47,9)$ & $153(41,2)$ \\
\hline \multicolumn{4}{|l|}{ Tipo de tratamento } \\
\hline Necropulpectomia & $351(84,8)$ & $103(83,1)$ & $248(85,5)$ \\
\hline Biopulpectomia & $52(12,6)$ & $13(10,5)$ & $39(13,4)$ \\
\hline Retratamento & $11(2,7)$ & $8(6,5)$ & $3(1)$ \\
\hline Outros & $2(0,4)$ & $0(0)$ & $2(0,5)$ \\
\hline \multicolumn{4}{|l|}{ Presença de dor } \\
\hline Sim & $140(26)$ & $47(28,1)$ & $93(25,1)$ \\
\hline Não & $398(74)$ & $120(71,9)$ & $278(74,9)$ \\
\hline \multicolumn{4}{|l|}{ Necessidade de adequação } \\
\hline Sim & $188(34,9)$ & $54(32,3)$ & $134(36,1)$ \\
\hline Não & $350(65,1)$ & $113(67,7)$ & $237(63,9)$ \\
\hline \multicolumn{4}{|c|}{ Impossibilidade de tratamento } \\
\hline Sim & $74(13,8)$ & $23(13,8)$ & $51(13,7)$ \\
\hline Não & $464(86,2)$ & $144(86,2)$ & $320(86,3)$ \\
\hline \multicolumn{4}{|l|}{ Número de sessões } \\
\hline Uma sessão & $9(2,5)$ & $1(9)$ & $8(3,2)$ \\
\hline Duas sessões & $100(28,1)$ & $25(23,1)$ & $75(30,2)$ \\
\hline Três sessões & $181(50,8)$ & $51(47,2)$ & $130(52,4)$ \\
\hline Quatro ou mais & $66(18,5)$ & $31(28,7)$ & $35(14,1)$ \\
\hline \multicolumn{4}{|l|}{ Tratamento concluído } \\
\hline Sim & $354(65,8)$ & $107(64,1)$ & $247(66,6)$ \\
\hline Não & $162(30,1)$ & $54(32,3)$ & $112(30,1)$ \\
\hline Não necessitou & $18(3,3)$ & $6(3,6)$ & $12(3,2)$ \\
\hline \multicolumn{4}{|l|}{ Abandono do tratamento } \\
\hline Sim & $92(17,1)$ & $33(19,8)$ & $59(15,9)$ \\
\hline Não & $428(79,6)$ & $128(76,6)$ & $300(80,9)$ \\
\hline Não necessitava & $18(3,3)$ & $6(3,6)$ & $12(3,2)$ \\
\hline
\end{tabular}




\section{DISCUSSÃO}

Foram encontradas diferenças entre as prevalências das variáveis em relação aos distritos sanitários para os pacientes e para os procedimentos. Em função da maioria das unidades básicas de saúde que referenciam usuários para o CEO Bom Jesus serem da gerência distrital Partenon-Lomba do Pinheiro, esta diferença pode explicar porque $68,8 \%$ dos prontuários deste estudo eram oriundos da gerência Partenon-Lomba do Pinheiro. Como um dos campos de estágio dos estudantes de Odontologia da Universidade Federal do Rio Grande do Sul é na Lomba do Pinheiro, os alunos têm a possibilidade de vivenciar as práticas de atenção básica à saúde relacionando-as com os dispositivos de referência e contrarreferência para os serviços de atenção especializada em saúde bucal, neste caso o CEO.

No presente estudo, 70,2\% dos usuários eram do sexo feminino, o que está em conformidade com os achados de Chaves et $\mathrm{al}^{17}$, que encontraram $68,1 \%$ e $70,3 \%$ de mulheres em dois municípios no estado da Bahia e de Saliba et $\mathrm{al}^{18}$, que encontraram $61,5 \%$ de pacientes do gênero feminino. Porém os dados dos estudos de Saliba et $\mathrm{al}^{18}$ e Chaves et $\mathrm{al}^{17}$, referem-se a usuários encaminhados para todas as especialidades do CEO. Esta maior prevalência do sexo feminino é um resultado comum nestes tipos de trabalho, seja da área médica ou odontológica, pois mulheres apresentam maior interesse no cuidado com a saúde, por terem mais autopercepção de saúde que os homens, resultando na maior busca por esses serviços ${ }^{19}$.

Os dentes que foram mais frequentemente tratados nas pessoas que utilizaram o serviço foram os primeiros molares $(38,5 \%)$, seguido de pré-molares $(22,7 \%)$, incisivos $(19,1 \%)$, segundos molares (13\%) e caninos (5,8\%). Pereira, Carvalho $^{20}$, avaliando prontuários de pacientes que foram tratados endodonticamente nas clínicas odontológicas do Centro Universitário de Lavras (MG), encontraram os seguintes resultados: para o sexo feminino, molar superior (25\%), incisivo superior e molar inferior $(19,4 \%)$, pré-molar superior $(16,1 \%)$, pré-molar inferior $(8,4 \%)$. Já para o sexo masculino, encontraram molar superior (24\%), incisivo superior (24\%), molar inferior $(23 \%)$, pré-molar superior $(11 \%)$, prémolar inferior (5\%). A explicação para os molares serem os dentes mais acometidos pode se relacionar ao fato de estarem em uma posição mais posterior no arco, tendo sua higiene dificultada, acumulando mais placa. Por outro lado, o grande número de incisivos pode estar relacionado aos traumatismos ocorridos, que geralmente afetam os dentes anteriores ${ }^{20}$. Sabe-se que os primeiros molares são os dentes mais acometidos por lesões cariosas, pois a atividade cariogênica inicia-se no seu longo período de erupção. Ocorre uma maior retenção de placa, prolongando-se esta situação até os dentes entrarem em oclusão, ao redor dos oito anos de idade ${ }^{21}$. Este pode ser o motivo destes dentes representarem uma grande prevalência de casos no presente estudo.

$\mathrm{O}$ tipo de tratamento mais indicado foi a necropulpectomia $(84,8 \%)$ seguido da biopulpectomia $(12,6 \%)$. Uma pesquisa realizada nas clinicas de um curso de Odontologia na cidade de Lavras (MG) que necropulpectomia com lesão e retratamentos representaram $42,8 \%$ dos casos no sexo feminino e $50 \%$ no sexo masculino. A segunda maior prevalência foi representada pela biopulpectomia $(31,3 \%$ e $27 \%)$, seguida pela necropulpectomia $(14,5 \%$ e $11,4 \%)$ para o sexo feminino e masculino, respectivamente ${ }^{20}$.

Durante as consultas para realização do tratamento endodôntico, a dor foi relatada em $26 \%$ dos casos. Este dado deve ser também considerado sob a perspectiva de que a dor está intimamente ligada à insatisfação do usuário com o tratamento endodôntico. Sua ausência é uma das principais expectativas dos usuários ao iniciarem o tratamento endodôntico ${ }^{22}$.

O protocolo de atenção em saúde bucal de Porto Alegre ${ }^{16}$ prevê uma série de critérios clínicos e de exclusão que devem ser considerados antes de os pacientes serem encaminhados para especialidade de Endodontia no CEO. Porém, o 
presente estudo apontou que 34,9\% dos dentes referenciados necessitavam de adequação para realização do tratamento endodôntico e 13,8\% foram considerados com impossibilidade de tratamento endodôntico. Os motivos são diversos, e vão desde a não remoção total de tecido cariado, até a ausência do elemento dentário em boca. Estes resultados evidenciam que existem lacunas em parte dos encaminhamentos, diferenças entre o que é preconizado e o que é realizado nas unidades de atenção primária que referenciam para os CEOS. Este fato reforça a importância da vivência de estudantes de Odontologia nos estágios de saúde pública e de aspectos relacionados ao processo de trabalho das equipes de atenção primária e dos CEO, pois é através da discussão e ressignificação das práticas de saúde que o ensino pode ser desenvolvido em caráter crítico-reflexivo.

O resultado mostrou que $81,5 \%$ dos tratamentos endodônticos foram concluídos em até três sessões, sendo que a primeira consulta, geralmente é utilizada para anamnese, exame clínico e radiografia inicial, e apenas na segunda sessão, o tratamento endodôntico propriamente dito é iniciado. Saliba et al ${ }^{18}$ calcularam em média 4,1 consultas necessárias para o tratamento endodôntico. Entretanto, a consulta de conclusão do caso na atenção primária foi contabilizada neste cálculo, diferentemente do presente estudo, que contabilizou apenas as sessões no CEO.

A porcentagem de tratamentos concluídos no presente estudo foi de $65,8 \%$. Este resultado é semelhante ao de Saliba et $\mathrm{al}^{18}$, que encontraram uma percentagem de $69,58 \%$ de tratamentos concluídos na especialidade de endodontia, porém $10,68 \%$ do total ainda estava com o tratamento em andamento. Já Bulgareli et $\mathrm{al}^{6}$ encontraram uma porcentagem de resolutividade na especialidade de endodontia de $50 \%$ para usuários referenciados de unidades básicas de saúde e 71,4\% de unidades de saúde da família, no município de Marília, SP. O número elevado de tratamentos não concluídos em nosso estudo pode ter diversas explicações. Entre elas o alto índice de necessidade de adequação do dente $(34,9 \%)$, abandono do tratamento $(17,1 \%)$, impossibilidade de tratamento $(13,8 \%)$, e ainda dentes que não necessitavam de tratamento endodôntico $(3,3 \%)$. Neste sentido, Vasquez et al ${ }^{2}$ avaliaram o sistema de referência e contrarreferência e o processo de trabalho na atenção secundária em saúde bucal em duas Regiões Administrativas de Saúde no município de Campinas, SP. Os autores evidenciaram problemas relacionados ao processo de trabalho, tais como a desorganização e a precariedade dos arquivos, cujas consequências vão desde a dificuldade de contato com o usuário para eventuais avisos, até a impossibilidade de planejamento da oferta, uma vez que a falta de informações compromete o reconhecimento real das necessidades. Concluíram que o processo de trabalho foi $\mathrm{o}$ fator preponderante para a explicação da resolutividade. Rodrigues et $\mathrm{al}^{3}$ também avaliaram este sistema de referência e contrarreferência, em um CEO na cidade de Juiz de Fora, MG e calcularam a porcentagem de prontuários que estavam em conformidade com os critérios preconizados (legibilidade, completude, indicação conforme critérios do MS). Os resultados obtidos foram $71,4 \%$ de prontuários completos para endodontia, $62,9 \%$ para cirurgia e diagnóstico de câncer bucal e $74 \%$ para periodontia; $74,3 \%$ classificados como legíveis para endodontia, $72 \%$ para cirurgia e diagnóstico de câncer bucal e 84,3\% para Periodontia. Havia indicação de tratamento especializado em $62,1 \%$ dos prontuários para endodontia, 59,4\% para cirurgia e diagnóstico de câncer bucal e 77,2\% para periodontia. Embora o presente estudo não tenha abordado a qualificação profissional de quem realizou o atendimento, a inadequação do uso de protocolos remete à questão da educação permanente e da formação de tais profissionais. Martins et $\mathrm{al}^{23}$ avaliaram o sistema de referência e contrarreferência em endodontia, na cidade de Criciúma, SC e compararam encaminhamentos feitos por dentistas com pós-graduação em residência multiprofissional e outros sem especialização na área. $\mathrm{O}$ resultado mostrou que o sistema de encaminhamentos foi resolutivo e que 
$82,4 \%$ dos pacientes que foram encaminhados pelos pós-graduados concluíram o tratamento, contra $60,9 \%$ dos encaminhados por dentistas sem pós-graduação. Os autores concluíram que o tratamento foi resolutivo quando o paciente foi bem orientado e realmente seguiu as orientações do profissional, comprovando a importância da educação em saúde na efetividade das ações.

A porcentagem de abandono do tratamento encontrada neste estudo foi considerada alta $(17,1 \%)$ cerca de três vezes maior do que a observada em estudo realizado na cidade de Marília, SP (6,21\% para usuários referenciados de unidades de atenção básica) ${ }^{6}$. Já outro estudo registrou um percentual de evasão, na especialidade de Endodontia, de 30,42\%. Os autores justificaram, afirmando que o tratamento endodôntico, por ser longo e muitas vezes procurado apenas para resolver a dor, acarretaria na desistência do paciente ao restante do tratamento. Ainda, os autores sugeriram a necessidade de conscientização do paciente, por meio de uma estratégia de educação em saúde, como forma de diminuir a evasão ao tratamento ${ }^{18}$.

Para Rocha, Bercht ${ }^{24}$, após analisar o abandono ao tratamento no Centro de Saúde Murialdo em Porto Alegre, RS, as principais causas foram a dificuldade em faltar ao trabalho, longo tempo de duração do tratamento, doenças, gravidez e mudança de domicílio, o que também poderia explicar o percentual de abandono encontrado no presente estudo.

Como limitação do estudo, o fato de não se ter a informação sobre tempo de espera para o atendimento não possibilita a avaliação do percurso/trajetória do usuário entre os níveis de atenção em saúde, fato que possui implicações nas condições clínicas no momento do atendimento no CEO, além de poder influenciar no absenteísmo. Outra limitação está no fato de não terem sido avaliadas as características socioeconômicas dos indivíduos, pois as variáveis estudadas abrangeram apenas informações coletadas nos prontuários. Porém, sabe-se que os determinantes sociais agem como fatores de risco para as doenças bucais, entre elas a cárie, que está intimamente ligada à necessidade de endodontia e à perda dentária ${ }^{25,26}$.

Uma das formas de se avaliar as características sociais dos indivíduos é através do índice de desenvolvimento humano (IDH) das regiões em que vivem. Ao avaliar as regiões pertencentes às gerencias distritais objetos de estudo do presente trabalho, constata-se grande discrepância entre os distritos, sendo que as regiões Leste e Partenon são mais desenvolvidas que as outras duas ${ }^{13}$.

Outro dado importante é a distribuição de domicílios indigentes e pobres. Novamente a região mais afetada é a nordeste com $9,6 \%$ de domicílios indigentes (DI) e 32,36\% de domicílios pobres (DP), seguida das regiões Lomba do Pinheiro 5,23\% (DI) e 22,56\% (DP), Partenon $3,15 \%$ (DI) e $13,31 \%$ (DP), Leste $2,83 \%$ (DI) e $12,09 \%(\mathrm{DP})^{13}$.

O planejamento no SUS deve ser baseado em dados epidemiológicos e demográficos que expressem as reais necessidades de saúde da população, e não apenas levar em consideração parâmetros construídos com base em números relativos à produção.

\section{CONCLUSÃO}

A análise realizada no CEO Bom Jesus permitiu delinear o perfil dos atendimentos em Endodontia. Foi possível identificar o distrito sanitário de maior demanda para o serviço, bem como características demográficas como sexo e idade. Além disso, a identificação de características relacionadas aos procedimentos poderá auxiliar no planejamento e resolutividade das ações, prevendo a demanda de atenção em saúde, onde através de estratégias de educação permanente, essas questões poderão ser discutidas com objetivo de qualificar o processo de trabalho das equipes de atenção primária, dos profissionais do CEO e dos estudantes de Odontologia favorecendo uma formação mais próxima do cotidiano a ser vivenciado no exercício profissional. 


\section{ABSTRACT \\ Evaluation of endodontic procedures in a Secondary Dental Care Center in Porto Alegre, $R S$}

This study aimed to describe the profile of endodontic procedures at Bom Jesus Secondary Dental Care Center. This is a descriptive crosssectional study using clinical records of patients treated between 2012 and 2013, in the specialty of endodontics, from two health districts of Porto Alegre. Absolute and relative frequencies of variables related to patients and procedures were carried out by general aspects and health district. The majority of users $(70.2 \%)$ were female, aged up to 19 years $(22.2 \%)$ and from the Parthenon district $(68.8 \%)$. The first molars showed highest demand $(38.5 \%)$ followed by premolars $(22.7 \%)$, as well teeth of upper arch $(56.7 \%)$ and necropulpectomia $(84.8 \%)$. In conclusion, it was possible to identify the health district of highest demand, as well as demographic and clinical characteristics of the patients, helping on planning and resolution of health service actions on National Health System (SUS) and in dental undergraduate education closest to the practice.

Descriptors: Dental Health Services. Secondary Care. Dental Specialties. Endodontics.

\section{REFERÊNCIAS}

1. da Luz GW, Toassi RFC. Percepções sobre o preceptor cirurgião-dentista da Atenção Primária à Saúde no ensino da Odontologia. Rev ABENO 2016; 16(1):2-12.

2. Vazquez FL, Guerra LM, Vítor ES, Ambrosano GMB, Mialhe FL, Meneguim MC, et al. Referência e contrarreferência na Atenção Secundária em Odontologia em Campinas, SP, Brasil. Ciênc Saúde Coletiva 2014; 19(1):24555.

3. Rodrigues LA, Vieira JDM, Leite ICG. Avaliação do fluxo de referência para um centro de especialidades odontológicas implantado em cidade de médio porte na Região Sudeste. Cad Saúde Colet 2013; 21(1): 40-5.
4. Mendes EV, Teixeira CF, Unglert CVS, Grego C, Araújo EC, Villarosa FN, et al. Distrito sanitário: o processo social de mudança das práticas sanitárias do SUS. $3^{a}$ ed. São Paulo: Hucitec-abrasco; 1995.

5. Moretto A, Santos AM, Aguinsky BG, Barcellos C, Ballester DA, Harzheim E, et al. Diagnóstico de saúde do distrito leste de Porto Alegre. Porto Alegre: EDIPUCRS; 2005.

6. Bulgareli JV, Faria ET, Ambrosano GMB, Vazquez FL, Cortellazzi KL, Meneghim MC, et al. Informações da atenção secundária em odontologia para avaliação dos modelos de atenção à saúde. Rev Odontol UNESP 2013; 42(4): 229-36.

7. Brasil. Ministério da Saúde. Brasil Sorridente, Centro de Especialidades Odontológicas. Acesso em 10 de junho de 2015. Disponível em: http://dab.saude.gov.br/portaldab/ape_bra sil_sorridente.php?conteudo=ceo

8. Brasil. Ministério da Saúde. Secretaria de Atenção à Saúde. Departamento de Atenção Básica. Coordenação Nacional de Saúde Bucal. Diretrizes da Política Nacional de Saúde Bucal. Brasília, 2004. Acesso em 10 de junho de 2015. Disponível em: http://bvsms.saude.gov.br/bvs/ publicacoes/politica_nacional_brasil_sorrident e.pdf

9. Brasil. Ministério da Saúde. Portaria n. 1341/GM, 13 de junho de 2012. Define os valores dos incentivos de implantação e de custeio mensal dos Centros de Especialidades Odontológicas - CEO e dá outras providências. Brasília, 2012.

10. Figueiredo N, Goes PSA. Construção da atenção secundária em saúde bucal: um estudo sobre os Centros de Especialidades Odontológicas em Pernambuco, Brasil. Cad Saúde Pública 2009; 25(2):259-67.

11. Goes PSA, Figueiredo N, Neves JC, Silveira FMM, Costa JFR, Júnior GAP, et al. Avaliação da atenção secundária em saúde bucal: uma investigação nos centros de especialidades do Brasil. Cad Saúde Pública 2012; 28(Supl):S819. 
12. Herkrath FJ, Herkrath APCQ, Costa LNBS, Gonçalves MJF. Desempenho dos Centros de Especialidades Odontológicas frente ao quadro sociodemográfico dos municípios do Amazonas, Brasil, 2009. Saúde Debate 2013; 37(96):148-58.

13. Porto Alegre. Plano Municipal de Saúde 2014 2017. Acesso em 10 de junho de 2015. Disponível em: http://lproweb.procempa.com.

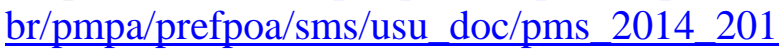
7.pdf

14. Porto Alegre. Centro de Saúde Bom Jesus. Acesso em 10 de junho de 2015. Disponível em: http://www2.portoalegre.rs.gov.br/sms/ default.php?reg=2\&p_secao $=835$

15. Brasil. Ministério da Saúde. Secretaria de Atenção à Saúde. Departamento de Atenção Básica. Manual de Especialidades em Saúde Bucal. Brasília, 2008.

16. Porto Alegre. Secretaria Municipal de Saúde. Área Técnica de Saúde Bucal. Protocolo de atenção em saúde bucal de Porto alegre: Organização da rede e fluxos de atendimento. Porto Alegre (RS); 2014; pg 42, 44, 45,46.

17. Chaves SCL, Soares FF, Rossi TRA, Cangussu MCT, Figueiredo ACL, Cruz DN, et al. Características do acesso e utilização de serviços odontológicos em municípios de médio porte. Ciênc Saúde Coletiva 2012; 17(11): 3115-24.

18. Saliba NA, Nayme JGR, Moimaz SAS, Cecilio LPP, Garbin CAS. Organização da demanda de um Centro de Especialidades Odontológicas. Rev Odontol UNESP 2013; 42(5):317-23.

19. Pinheiro RS, Torres TZG. Uso de serviços odontológicos entre os Estados do Brasil. Ciênc Saúde Coletiva 2006; 11(4): 999-1010.

20. Pereira CV, Carvalho JC. Prevalência e eficácia dos tratamentos endodônticos realizados no Centro Universitário de Lavras, MG - uma análise etiológica e radiográfica. RFO 2008; 13 (3):36-41.

21. Carvalho JC, Ekstrand KR, Thylstrup A. Dental plaque and caries on occlusal surfaces of first permanent molars in relation to stage of eruption. J Dent Res 1989; 68(5):773-79.

22. Pontes, ALB. Avaliação da satisfação do usuário e da qualidade dos tratamentos endodônticos em Centros de Especialidades Odontológicas da Grande Natal - RN. [Dissertação]. Natal: Universidade Federal do Rio Grande do Norte; 2011.

23. Martins LGT, Traebert J, Sônego FGF. Análise do sistema de referência em contra-referência em um serviço público de odontologia. Rev Saúde Públ Santa Cat 2013; 6(4):6-18.

24. Rocha CR, Bercht, SB. Estudo do abandono do tratamento odontológico de um serviço público de Porto Alegre: o Centro de Saúde Murialdo. Rev Fac Odontol Porto Alegre 2000; 42 (2):2531.

25. Peres MA, MRDO Latorre, Sheimam A, Peres KG, Barros FC, Hernandez PG, et al. Determinantes sociais e biológicos da cárie dentária em crianças de 6 anos de idade: um estudo transversal aninhado numa coorte de nascidos vivos no Sul do Brasil. Rev Bras Epidemiol 2003; 6(4):293-306.

26. Antunes JLF, Peres MA, Mello TRC. Determinantes individuais e contextuais da necessidade de tratamento odontológico na dentição decídua no Brasil. Ciênc Saúde Coletiva 2006; 11(1):79-87.

Correspondência para:

Jessye Melgarejo do Amaral Giordani

e-mail: giordanijma@gmail.com

Av. Roraima, 1000 - Campus UFSM

Prédio 26, $3^{\circ}$ Andar, Sala 1356 - Camobi

97105-900, Santa Maria/RS 